\title{
Effect of Buffer Gas on the Fluorescence Yield of Trapped Gas-Phase Ions
}

\author{
Maxim Dashtiev and Renato Zenobi \\ Department of Chemistry and Applied Biosciences, ETH Zurich, Zurich, Switzerland
}

We investigated the dependence of three different gases, helium, argon, and nitrogen, on the fluorescence signal intensity of rhodamine $6 \mathrm{G}$ cations in the gas phase. The method is based on laser-induced fluorescence of ions trapped in a Fourier transform ion cyclotron mass spectrometer. We found that the use of helium results in the highest fluorescence signal, while no fluorescence was detected when using argon under the same conditions. (J Am Soc Mass Spectrom 2006, 17, 855-858) (c) 2006 American Society for Mass Spectrometry

$\mathrm{I}$ nformation on the structure and dynamics of biomolecules in the gas-phase environment is one of the ultimate goals of mass spectrometry. The possibility to isolate and generate biomolecular ions without solvent, salt, or any other adducts renders mass spectrometry very attractive. Different mass spectrometric approaches such as blackbody infrared radiative dissociation [1], hydrogen-deuterium exchange [2], covalent or noncovalent tagging of biomolecules [3, 4], collisioninduced dissociation [5], and ion mobility measurements [6] have been used to study the structure of molecules in the gas phase. Recently, a technique developed by Parks and coworkers [7-10], based on laser-induced fluorescence (LIF) of ions trapped in a modified Paul trap, has been successfully applied to probe conformational changes of oligonucleotides and proteins in the gas phase. In our laboratory, we adapted a Fourier transform ion cyclotron resonance (FT ICR) mass spectrometer for fluorescence measurements of trapped ions [11, 12]. FT ICR uses a Penning trap, which provides both temporal and spatial confinement of the ions and is, thus, well suited for studies combining optical and mass spectroscopic techniques. An FT ICR mass spectrometer has several advantages over an ion trap: higher resolution, easier manipulation of the ion cloud, and superior $\mathrm{MS}^{\mathrm{n}}$ capabilities. Several works were reported on fluorescence spectroscopy in an FT ICR mass spectrometer as well as in ion traps [7-17]. One similar feature for all fluorescence measurements, both in ion traps and FT ICR mass spectrometers, is that the fluorescence detection is accomplished in the presence a buffer gas. It is, thus, important to understand the effect of this buffer gas on the fluorescence signal intensity. The presence of a buffer gas is also necessary

Published online April 17, 2006

Address reprint requests to Professor Renato Zenobi, Department of Chemistry and Applied Biosciences, ETH Zurich, HCI E 329, CH-8093 Zurich, Switzerland. E-mail: zenobi@org.chem.ethz.ch for collisional cooling and spatial confinement of the ions. In ion traps, for example, the high amplitude rf voltage applied to the ring electrode forms a pseudopotential well with the minimum energy at the center of the trap; thus, collisions help to bring ions to its center. It has been demonstrated that the most efficient gas for cooling ions in a quadrupole ion trap is a light gas such as $\mathrm{He}$ or $\mathrm{H}_{2}$ [18]. For successful fluorescence measurements in an FT ICR it is necessary to have a very tight ion packet, to get a maximum overlap with the excitation laser. To achieve this in an ICR, quadrupolar axialization is used. An excellent review about quadrupolar axialization can be found in [19]. Various gases $\left(\mathrm{N}_{2}\right.$, Ar, He) are commonly used for quadrupolar axialization in FT ICR MS. We are not aware of any work that clearly states which gas provides the best quadrupolar axialization for FT ICR, which means as tight (compact) an ion cloud as possible, right after the axialization event. It will, thus, be important to choose a buffer gas so one could satisfy both criteria, optimum quadrupolar axialization, and highest fluorescence yield. Here we report on a study on the effect of three different gases $\left(\mathrm{Ar}, \mathrm{He}, \mathrm{N}_{2}\right.$ ) on the fluorescence yield of trapped rhodamine $6 \mathrm{G}$ ions.

\section{Experimental}

Rhodamine 6G was purchased from Acros Organics/ Chemie Brunschwig AG (Basel, Switzerland). The full description of the instrument is given elsewhere [12]. Briefly, the experiments were performed on a Fouriertransform ion cyclotron resonance (FT ICR) mass spectrometer that consists of a home-built vacuum system, a 4.7 T superconducting magnet (Bruker, Fällanden, Switzerland), and commercial control electronics and data acquisition (IonSpec Corp., Lake Forest, CA). For laser desorption/ionization, the third harmonic of Nd:YAG laser (Minilite ML-10, Continuum; 5 ns pulse width; $\lambda=$ $355 \mathrm{~nm}$, Santa Clara, CA) was used. The fluorescence 


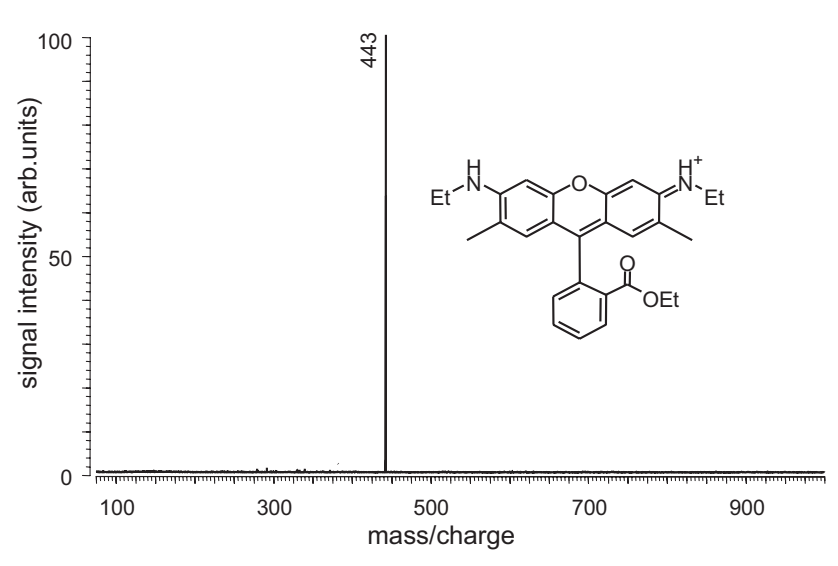

Figure 1. Mass spectrum of rhodamine 6G. The signal represents the average of 10 scans.

was collected in a similar fashion as in [11]. The trapped ions were excited by an Ar ion laser (Innova 300, Coherent, Santa Clara, CA) with $488 \mathrm{~nm}$. The Ar-laser diameter was $4 \mathrm{~mm}$ and the laser power for all experiments was $120 \mathrm{~mW}$. The emitted fluorescence was focused onto a collimator through a wire mesh covered cut-out in one of the cell plates. The collimator focused the light onto an optical fiber, which is connected to an optical vacuum feedthrough (Caburn Vacuum Science, Ltd., Glynde/East Sussex, UK) followed by an additional optical fiber that focused the light to another collimator, then to a long pass filter (FEL 500, Thorlabs, Karlsfeld, Germany), and finally to the detector (SPCMACQ-16, PerkinElmer, Fremont, CA). The signal is recorded by an $800 \mathrm{MHz}$ gated single photon counter/multiscaler (PMS 400, Becker and Hickl $\mathrm{GmbH}$, Berlin, Germany). The fluorescence signal represents the averaged fluorescence from 10 scans. Buffer gas was introduced via a leak valve for $10 \mathrm{~s}$ to a pressure of $10^{-3}$ mbar with simultaneous quadrupolar axialization of the ions for $19 \mathrm{~s}$, followed by chirp excitation and detection. The laser for exciting rhodamine 6G ions was on during the whole sequence (20 s). Since our vacuum gauge is calibrated for nitrogen, calibration factors for other gases were applied to maintain the same absolute pressure. MALDI samples were prepared using a "tablet" method using 2,5- dihydroxybenzoic acid (DHB, Acros Organics) as a matrix. By "tablet" we mean the following sample preparation method: first, rhodamine $6 \mathrm{G}$ was dissolved in methanol at a $10^{-3} \mathrm{M}$ concentration; then dry DHB was placed on the target and slightly pressed, and finally rhodamine 6G was dropped onto it. To determine the error bars of the data, one standard deviation from the mean was calculated. Ten measurements from different spots were used to calculate the deviation. The calculated standard deviation was about $13 \%$ for all three gases. The calculated standard deviation for the fluorescence signal was around $5 \%$, i.e., the total error was about $14 \%$.

\section{Results and Discussion}

Figures 1 and 2 show the mass spectrum and the corresponding fluorescence signals from rhodamine 6G, when using different buffer gases (Figure 2a) helium, (Figure 2b) nitrogen, and (Figure 2c) argon. The fluorescence signal when using He is $160 \pm 22$ photons/s, for $\mathrm{N}_{2}$ it is $105 \pm 15$ photons/s, and no fluores-

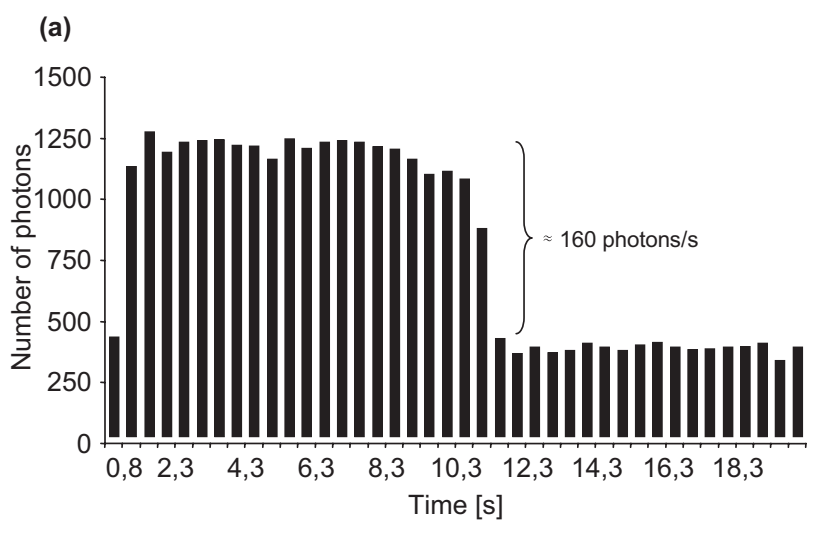

(b)

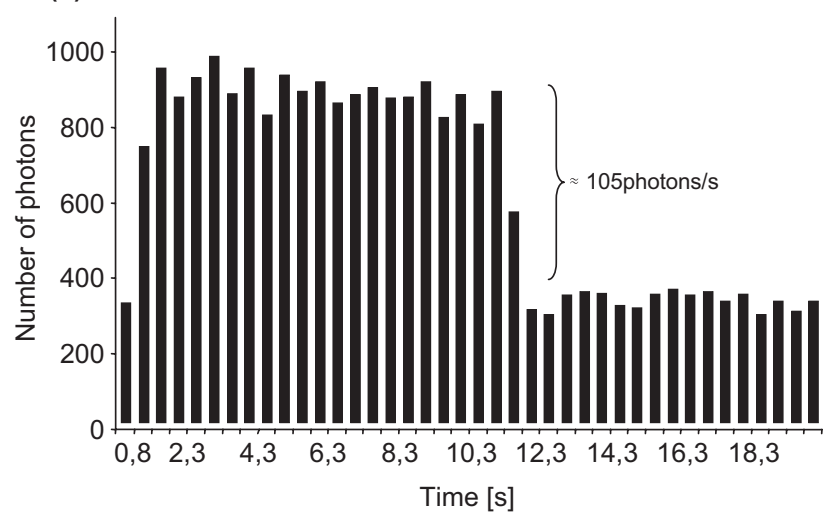

(c)

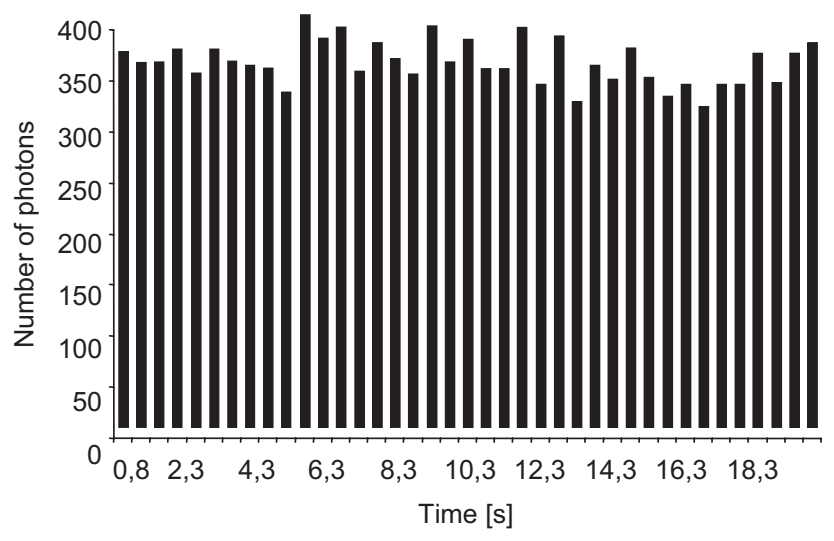

Figure 2. Dependence of the fluorescence signal intensity of rhodamine $6 \mathrm{G}$ ions versus time on three different gases, (a) helium, (b) nitrogen, and (c) argon. The excitation wavelength was $488 \mathrm{~nm}$ and the laser power was $120 \mathrm{~mW}$. The rapid increase of the fluorescence is due to the pressure increase of the buffer gas in the trap region. The background signal represents the number of photons reaching the detector after the pressure is brought back to a nominal value of $\leq 10^{-7}$ mbar. 
cence is observed for Ar. Let us now consider possible scenarios for these dramatically different fluorescence signal levels.

\section{Difference in Spatial Overlap Due to Efficiency of Quadrupolar Axialization}

Quadrupolar axialization can affect the dimension of the ion cloud in two ways: (1) contraction of the ion cloud in radial direction, perpendicular to the magnetic field vector, and (2) ion cloud compression in $\mathrm{z}$-direction, parallel to the magnetic field vector. The ion cloud compression in z-direction is mainly due to collisional cooling by the neutral buffer gas. Typical times for this cooling are about milliseconds. The radial shrinking is due to an azimuthal radiofrequency field applied that periodically converts magnetron motion to cyclotron motion, while cyclotron motion is damped with collisions [19]. This process can require between milliseconds up to a few seconds. The strong increase in fluorescence signal during the first $2 \mathrm{~s}$ after starting axialization shows the effect of spatial compression of the ion cloud both in radial and in z-direction (Figure $2 \mathrm{a}, \mathrm{b}$ ). Thus we believe that after $\sim 2 \mathrm{~s}$ we obtain an ion packet of approximately the same size no matter which gas is used $\left(\mathrm{N}_{2}, \mathrm{Ar}, \mathrm{He}\right)$. The MS amplitude of the ion signal and the resolution were the same for all three gases, which is also a clear indication that the size of the ion packet is very similar. Thus, we do not expect any difference in fluorescence signal intensity due to quadrupolar axialization geometric effects.

\section{Quenching}

One effect that may be responsible for fluorescencequenching is related to the polarizability of Ar. The polarizability of $\mathrm{Ar}$ is $1.85 \AA^{3}$, which is much higher than that of He $0.22 \AA^{3}$ [20]. This means that Ar atoms, which are in the vicinity of rhodamine $6 \mathrm{G}$ cations, are easily polarized. The charge-dipole interaction can result in the formation of noncovalent complexes of $\mathrm{Ar}$ with rhodamine $6 \mathrm{G}$ cations. This is not expected to occur for $\mathrm{He}$ at room temperature. One can think of several possible scenarios of how this should quench the fluorescence: (1) the quantum yield of the complex could be much lower than that for uncomplexed rhodamine $6 \mathrm{G}$ ions, or (2) formation of the complex may result in a shift of the optical absorption spectrum. We did not observe any MS signal from this complex, which indicates a short lifetime. The polarizability of $\mathrm{N}_{2}$ is 1.97 [20], which is slightly higher then that of Ar. Thus, one would expect the formation of the same kind of complexes with $\mathrm{N}_{2}$ as in case of Ar. This suggests that fluorescence-quenching with $\mathrm{N}_{2}$ should be even more pronounced. However, this is not observed, which argues against fluorescence-quenching by the formation of a buffer gas-rhodamine 6G complex.

\section{Intersystem Crossing}

Another effect, the so-called "external heavy atom effect" may affect the fluorescence yield of a collision complex during the following process: $\mathrm{M}^{*}+\mathrm{Ar} \rightarrow \mathrm{M}+$ $\mathrm{Ar}+$ heat.

It is beyond the scope of this study to fully describe the physical principle of the external heavy atom effect. A thorough explanation can be found in excellent books [21, 22]. However it does seem appropriate to give a brief overview. It has been shown that the presence of a heavy atom increases the spin-orbit coupling, i.e., coupling between the orbital magnetic moment and the spin magnetic moment. Thanks to this interaction, there is the probability for intersystem crossing (i.e., a forbidden transition from the first singlet excited-state $S_{1}$ to the first triplet state $T_{1}$ ). The efficiency of spin-orbit coupling varies with the fourth power of the atomic number, which explains why intersystem crossing is favored by the presence of heavy atoms. Thus, the probability of intersystem crossing for rhodamine $6 \mathrm{G}$ ions in contact with $\mathrm{Ar}$ is $10^{4}$ times higher compared to He. Once intersystem crossing has occurred, the main deactivation pathways are nonradiative decay, delayed fluorescence, and phosphorescence. Since we did not observe any delayed fluorescence. this pathway is not discussed here. It is known that in solution at room temperature, nonradiative decay from the triplet state $T_{1}$ is in general predominant over radiative de-excitation or phosphorescence [22]. The phosphorescence lifetime is in the range of $10^{-6} \mathrm{~s}$ to several seconds. To see whether we can observe any phosphorescence, we performed an additional experiment with the Arexcitation laser shut off after 100, 200, $500 \mathrm{~ms}$, and $1 \mathrm{~s}$. No phosphorescence was observed, indicating that either the phosphorescence lifetime for rhodamine 6G is less than $100 \mathrm{~ms}$ or there is only a nonradiative channel of energy dissipation. Unfortunately, we do not have a possibility to go below $100 \mathrm{~ms}$ since we have a minimum time uncertainty of $100 \mathrm{~ms}$ due to the opening/closing time of the shutter, which is used to block the laser.

\section{Conclusions}

The most probable explanation for the effect of the buffer gas on the fluorescence yield of trapped rhodamine 6G ions is the external heavy atom effect manifesting itself during ion-buffer gas collisions. Clearly, $\mathrm{He}$ is the most appropriate gas for performing fluorescence measurements in an FT ICR ion trap. Despite the fact that all three gases provide an efficient quadrupolar axialization, it is advantageous to use $\mathrm{He}$ since it provides the highest fluorescence signal, while axialization is as efficient as with $\mathrm{N}_{2}$ and He. We believe that this finding is of practical importance for performing future experiments involving fluorescence spectroscopy 
of ions in the gas phase both in ion traps and FT ICR traps.

\section{Acknowledgments}

The authors thank Dr. Mikhail Gorshkov for his helpful discussions.

\section{References}

1. Dunbar, R. C. BIRD (blackbody infrared radiative dissociation): Evolution, principles, and applications. Mass Spectrom. Rev. 2004, 23, 127-158.

2. Engen, J. R.; Smith, D. L. Investigating protein structure and dynamics by hydrogen exchange MS. Anal. Chem. 2001, 73, 256A-265A.

3. Friess, S. D.; Zenobi, R. Protein structure information from mass spectrometry? Selective titration of arginine residues by sulfonates. J. Am. Soc. Mass Spectrom. 2001, 12, 810-818.

4. Sharp, J. S.; Becker, J. M.; Hettich, R. L. Analysis of protein solvent accessible surfaces by photochemical oxidation and mass spectrometry. Anal. Chem. 2004, 76, 672-683.

5. Burlingame, A. L.; McCloskey, J. A. Biological Mass Spectrometry; Elsevier: Amsterdam, 1990; p 179.

6. Shelimov, K. B.; Clemmer, D. E.; Hudgins, R. R.; Jarrold, M. F. Protein structure in vacuo: Gas-phase conformations of BPTI and cytochrome $c$. J. Am. Chem. Soc. 1997, 119, 2240-2248.

7. Danell, A. S.; Parks, J. H. Fraying and electron autodetachment dynamics of trapped gas phase oligonucleotides. J. Am. Soc. Mass Spectrom. 2003, 14, 1330-1339.

8. Danell, A. S.; Parks, J. H. FRET. Measurements of trapped oligonucleotide duplexes. Int. J. Mass Spectrom. 2003, 229, 35-45.

9. Khoury, J. T.; Rodriguez-Cruz, S. E.; Parks, J. H. Pulsed fluorescence measurements of trapped molecular ions with zero background detection. J. Am. Soc. Mass Spectrom. 2002, 13, 696-708.

10. Iavarone, A. T.; Parks, J. H. Conformational change in unsolvated Trp-cage protein probed by fluorescence. J. Am. Chem. Soc. 2005, 127, 8606-8607.
11. Dashtiev, M.; Azov, V.; Frankevich, V.; Scharfenberg, L.; Zenobi, R. Clear evidence of fluorescence resonance energy transfer in gas-phase ions. I. Am. Soc. Mass Spectrom. 2005, 16, 1481-1487.

12. Frankevich, V.; Guan, X.; Dashtiev, M.; Zenobi, R.Laser-induced fluorescence of trapped gas-phase molecular ions generated by internal source matrix-assisted laser desorption/ionization in a Fourier transform ion cyclotron resonance mass spectrometer. Eur. J. Mass Spectrom. 2005, 11, 475-482.

13. Cage, B.; Friedrich, J.; Little, R. B.; Wang, Y. S.; McFarland, M. A.; Hendrickson, C. L.; Dalal, N.; Marshall, A. G. Wavelength resolved laser-induced fluorescence emission of $\mathrm{C}_{6} \mathrm{~F}_{3} \mathrm{H}_{3}{ }^{+}$trapped in an ion cyclotron resonance cell. Chem. Phys. Lett. 2004, 394, 188-193.

14. Cage, B.; McFarland, M. A.; Hendrickson, C. L.; Dalal, N. S.; Marshall, A. G. Resolution of individual component fluorescence lifetimes from a mixture of trapped ions by laser-induced fluorescence/ion cyclotron resonance. J. Phys. Chem. A 2002, 106, 10033-10036.

15. Friedrich, J.; Fu, J. M.; Hendrickson, C. L.; Marshall, A. G.; Wang, Y. S. Time resolved laser-induced fluorescence of electrosprayed ions confined in a linear quadrupole trap. Rev. Sci. Instrum. 2004, 75, 4511-4515.

16. Li, G. Z. Vining, B. A.; Guan, S. Marshall, A. G. Laser-induced fluorescence of $\mathrm{Ba}^{+}$ions trapped and mass-selected in a Fourier transform ion cyclotron resonance mass spectrometer. Rapid Commun. Mass Spectrom. 1996, 10, 1850-1854.

17. Wang, Y.; Hendrickson, C. L.; Marshall, A. G. Direct optical spectroscopy of gas-phase molecular ions trapped and mass-selected by ion cyclotron resonance: Laser-induced fluorescence excitation spectrum of hexafluorobenzene $\left(\mathrm{C}_{6} \mathrm{~F}_{6}{ }^{+}\right)$. Chem. Phys. Lett. 2001, 334, 69-75.

18. Stafford, G. C.; Kelley, P. E.; Syka, J. E. P.; Reynolds, W. E.; Todd, J. F. J. Recent Improvements in and Analytical Applications of Advanced Ion Trap Technology. Int. J. Mass Spectrom. 1984, 60, 85-98.

19. Guan, S. H.; Kim, H. S.; Marshall, a. G.; Wahl, M. C.; Wood, T. D.; Xiang, $X$. Shrink-Wrapping an Ion Cloud for High-Performance FourierTransform Ion-Cyclotron Resonance Mass-Spectrometry. Chem. Rev. 1994, 94, 2161-2182.

20. Böttcher, C. J. F.; Bordewijk P. Theory of Electric Polarization; Elsevier: Amsterdam, 1978

21. McGlynn, S. P.; Azumi, T.; Kinoshita, M. Molecular Spectroscopy of the Triplet State; Prentice-Hall Inc.: Englewood Cliffs, NJ, 1969; p 40.

22. Valeur, B. Molecular Fluorescence; Wiley-VCH Verlag GmbH: Weinheim, Germany, 2001; p 72. 